\title{
"Il coraggio di essere giovani": un progetto sulla Fgci di Modena degli anni Ottanta
}

L'Istituto Storico di Modena promuove una ricerca sulla Fgci di Modena degli anni Ottanta con l'obiettivo di cominciare a riflettere sul decennio conclusivo della storia del Pci, un periodo di crisi profonda della cultura politica comunista che la storiografia non ha ancora affrontato in modo sistematico. Il caso modenese permette di osservare alcuni importanti elementi dell'esperienza della Fgci "rifondata”, come il nuovo approccio alla comunicazione politica e il rapporto con i movimenti sociali.

The Historical Institute of Modena is undertaking a research project about the Communist Youth Federation of Modena in the 1980s, with the goal of looking for new perspectives on the last decade of history of the Italian Communist Party. The case of Modena offers a privileged point of observation on some of the most important features of the politics of the "new" Youth Federation, like its approach to political communication and its relationship with the social movements.

\section{Introduzione: lo scopo del progetto}

«Ci sentiamo estranei a questi anni Ottanta, i più brutti della nostra vita» [Ignazi 1992, 29]. Sono le parole con cui nel 1987, dopo la grave sconfitta elettorale subita dal Pci alle politiche, un segretario di sezione toscano tracciava un bilancio di quello che sarebbe stato il decennio conclusivo della storia del partito. Il riferimento al senso di estraneità percepito dalla base è particolarmente significativo: questa semplice espressione di scoramento diventa indice non solo della serie di insuccessi a livello organizzativo ed elettorale che i comunisti avevano incontrato 
dal 1979 in avanti, ma soprattutto di un arresto in senso identitario e culturale, dell'apparente incapacità del partito di riconoscersi nel contesto delle trasformazioni che stavano attraversando il paese così come l'intero assetto geopolitico mondiale, e di esprimere una nuova progettualità adatta ad affrontarle.

Questo bilancio emotivo, d'altronde, non si discosta molto da quello che viene generalmente proposto in ambito storiografico a ormai venticinque anni di distanza dalla svolta occhettiana. C'è accordo tra gli studiosi nell'identificare gli anni Ottanta con la progressiva "atrofia" del comunismo italiano [Ginsborg 1998, 293] e, infine, con la sua inevitabile "eclissi”" [Crainz 2012, 163]. Per la storia del Pci si tratterebbe insomma, nella definizione di Marcello Flores e Nicola Gallerano, di un decennio "residuale" rispetto a processi che si erano di fatto già avviati nella seconda metà degli anni Settanta e che sarebbero poi precipitati con il 1989 e la conseguente decisione della dirigenza comunista di cambiare nome e simbolo al partito [Flores e Gallerano 1992, 258].

Se da un lato, quindi, abbiamo di fronte un periodo facilmente riassumibile in un quadro interpretativo generale di inarrestabile declino di una importante cultura politica e dell'organizzazione che ne era portatrice, dall'altro gli anni Ottanta del Pci rappresentano un oggetto relativamente recente e ancora non affrontato in modo sistematico dalla storiografia. In questo contesto, il progetto dedicato alla Federazione Giovanile Comunista promosso dall'Istituto Storico di Modena insieme all'associazione "Insieme per Angela" appare invece come un tentativo di cominciare a comporre un'immagine più precisa di questo decennio, che ne metta in luce le complessità. Il progetto è partito nel 2014 con un duplice obiettivo: in primo luogo quello di riordinare il fondo della Fgci provinciale, che ne testimonia l'attività dalla fine degli anni Settanta fino ai primi anni di vita della Sinistra Giovanile; in secondo luogo, a partire proprio da questi documenti, quello di avviare una ricerca mirata a ricostruire la specifica esperienza della Federazione giovanile modenese durante gli anni Ottanta. La prima fase del progetto, che si è concentrata soprattutto sul riordino dell'archivio, si è conclusa con l'inizio del 2015, mentre i risultati iniziali della ricerca saranno presentati a gennaio 2016 a Modena, durante la giornata che ogni anno l'Istituto Storico di Modena e l'associazione "Insieme per Angela" dedicano alla presentazione dei progetti seguiti nei dodici mesi precedenti. Questa seconda fase si concluderà poi con la pubblicazione di un catalogo cartaceo dei materiali più significativi provenienti dall'archivio. 


\section{Il Pci degli anni Ottanta e i giovani. La scelta autonoma della Fgci}

Il primo elemento rilevante a emergere da questo progetto è la scelta di porre una lente di ingrandimento sul rapporto tra mondo giovanile e Pci, che negli anni Ottanta divenne uno dei nodi più critici per il partito anche all'interno delle apparentemente immutabili "zone rosse" del centro-Italia. Basti pensare che se nel 1975 la fascia demografica compresa tra i 18 e i 25 anni era sovrarappresentata tra gli elettori del Pci del 13,3\% rispetto all'elettorato complessivo, nel 1987 sarebbe arrivata a essere sottorappresentata del 2,9\%: nonostante questo evidente processo di distacco sia ancora poco esplorato, viene solitamente considerato uno dei fattori che hanno pesato in modo più rilevante sul declino finale del Pci [Mannheimer 1990, 57].

D'altronde, il rapporto tra giovani e Partito comunista aveva incontrato diversi momenti difficili già dall'inizio degli anni Sessanta, quando erano progressivamente comparse nuove forme della partecipazione politica giovanile verso cui il partito aveva spesso dimostrato diffidenza e incomprensione. In particolare, il Pci fu notoriamente restio a instaurare un dialogo con i movimenti nati dal 1968, anche se proprio la lunga stagione di mobilitazione aperta dai movimenti portò poi, nell'arco dei successivi otto anni, a un consistente spostamento verso sinistra dell'elettorato più giovane, di cui i comunisti cominciarono a beneficiare già con le elezioni del 1972 [Barbagli e Corbetta 1980, 485]. Questa influenza positiva fu percepita anche a livello organizzativo, e, nonostante non si possa parlare di vera e propria "confluenza" dei movimenti nel Pci, il ciclo di proteste degli anni Settanta rappresentò indubbiamente il contesto di formazione dell'ultima grande nuova generazione di militanti comunisti, che raggiunse numeri paragonabili a quelli del dopoguerra [Hellman 1980; Lange, Irvin e Tarrow 1990]. Tuttavia, il Partito comunista si dimostrò infine incapace di consolidare il consenso ottenuto in questo periodo, e questo momento di scambio favorevole con le generazioni più giovani si arrestò bruscamente alla fine degli anni Settanta, in coincidenza con la più ampia crisi che aveva investito il Pci dopo il fallimento del progetto del compromesso storico.

Prevedibilmente, a soffrire di questo allontanamento fu soprattutto la Fgci, che tra la metà degli anni Settanta e il 1985 vide i propri iscritti dimezzarsi [Gundle 1995, 466]. Schiacciata tra il movimentismo della seconda metà degli anni Settanta, che si poneva in aperto conflitto con il Pci, e il generale calo della partecipazione tra i giovani italiani, la Federazione giovanile sembrava destinata a scomparire all'interno del partito, a cui rimaneva strettamente subordinata. Ep- 


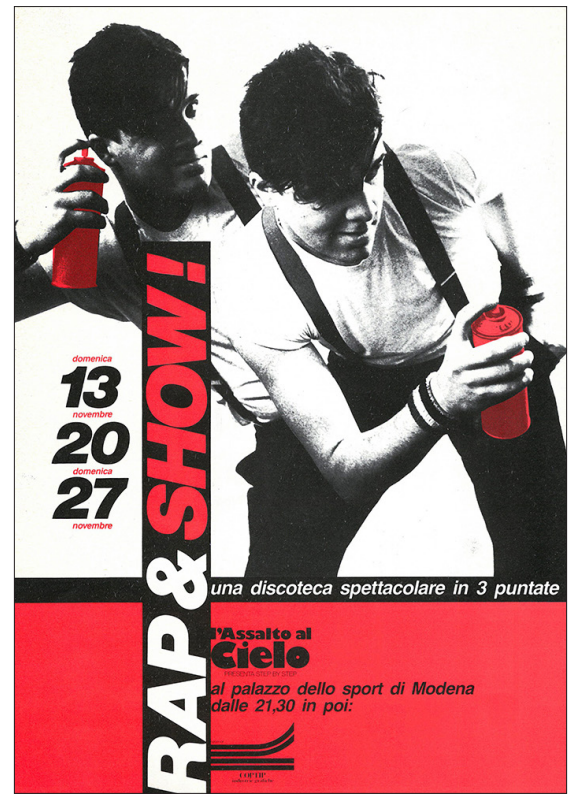

1984 - Locandina di "Rap\&Show", iniziativa organizzata da Kennedy's Studios e dalla redazione di " $L$ 'Assalto al Cielo", rivista della Fgci modenese. [art direction Elisabetta Ognibene/ Kennedy's studios] pure, nonostante la posizione minoritaria e le risorse limitate, fu proprio dalla Fgci che arrivò uno dei pochi segnali di reazione al momento di stallo che stavano attraversando i comunisti italiani. Già dal 1982 all'interno della Federazione si aprì un dibattito sul possibile rinnovamento dell'organizzazione che sfociò poi, con il Congresso di Napoli del 1985, nella decisione di proclamarne l'autonomia formale dal Pci, trasformandola in un'organizzazione federativa formata da sei "leghe" e "centri di iniziativa tematica" operanti in ambiti diversi: l'ambientalismo, il genere, i diritti civili, la pace, e così via?.

Era chiaro dunque il tentativo di avvicinarsi ai temi e ai linguaggi dei movimenti che si erano affacciati di recente sulla scena internazionale e di intraprendere una rifondazione non solo organizzativa, ma anche politica e ideologica. La parzia-

le rottura con il passato e la tradizione non fu completamente indolore, e i conflitti interni aperti da questa decisione affiorarono pubblicamente durante il Congresso di Napoli, dove Giancarlo Pajetta criticò esplicitamente quella che

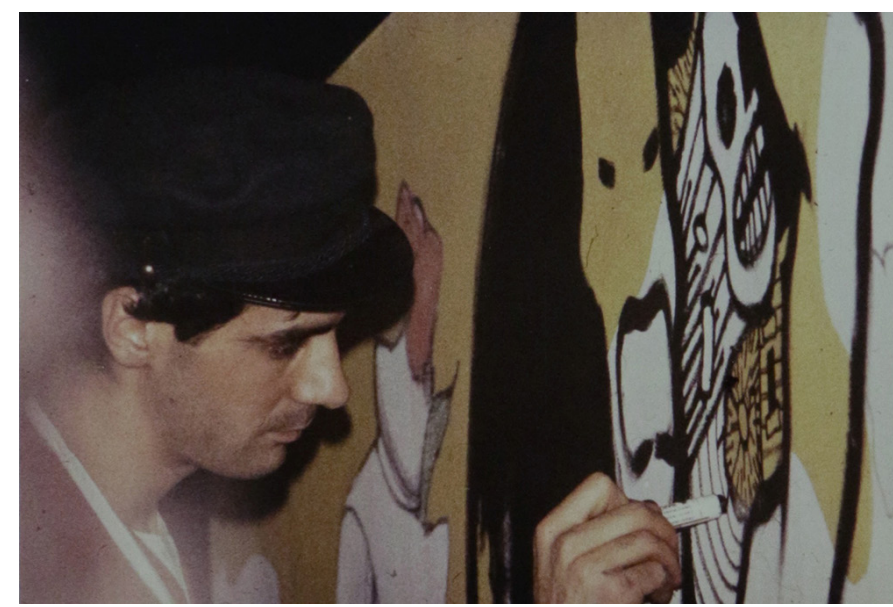

1984 - Andrea Pazienza disegna durante una delle serate di "Rap\&Show". [art direction Elisabetta Ognibene/Kennedy's studios] 
riteneva essere un'eccessiva influenza dei movimenti ecologisti e pacifisti sulle istanze avanzate dai giovani comunisti [Fotia 1985]. Tuttavia, l'intuizione della Fgci si rivelò infine corretta: mentre, paradossalmente, il Pci continuava a perdere voti e iscritti tra i giovani, i tesserati della Federazione giovanile rifondata e autonoma passavano dal minimo storico di 42.000 del 1985 a più di 50.000 nel 1988 [Mori 1988]. La Fgci, dunque, rappresentò un caso unico nel contesto di generale stasi che contraddistinse l'ultima fase della storia del Pci come partito di massa, ed è proprio questo lo scenario su cui sceglie di concentrarsi la ricerca promossa dall'Istituto Storico di Modena.

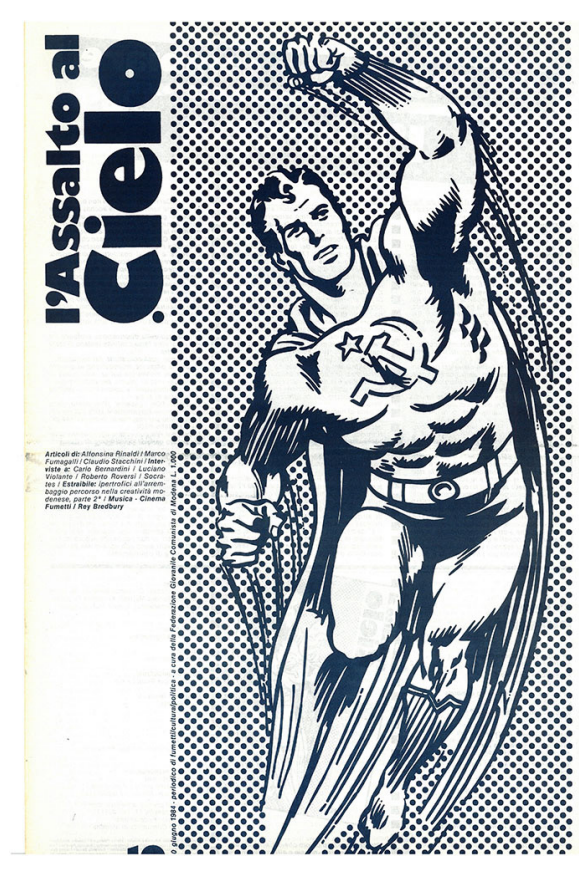

1984 - Copertina di "L'Assalto al Cielo", rivista della Fgci modenese. [art direction Elisabetta Ognibene/Kennedy's studios]

\section{Il caso di studio: la Fgci di Modena}

Il caso di Modena, in particolare, costituisce un punto di osservazione privilegiato per individuare gli elementi più significativi di questo processo di rinnovamento. Come testimonia la documentazione conservata nel fondo della Federazione provinciale, il cambiamento culturale e politico di cui la "nuova" Fgci cominciava a discutere all'inizio degli anni Ottanta si tradusse all'interno della Federazione modenese in un'interessante sperimentazione di nuove forme e strumenti della comunicazione politica, alla base della quale c'era la stretta collaborazione tra la dirigenza comunista e un gruppo di

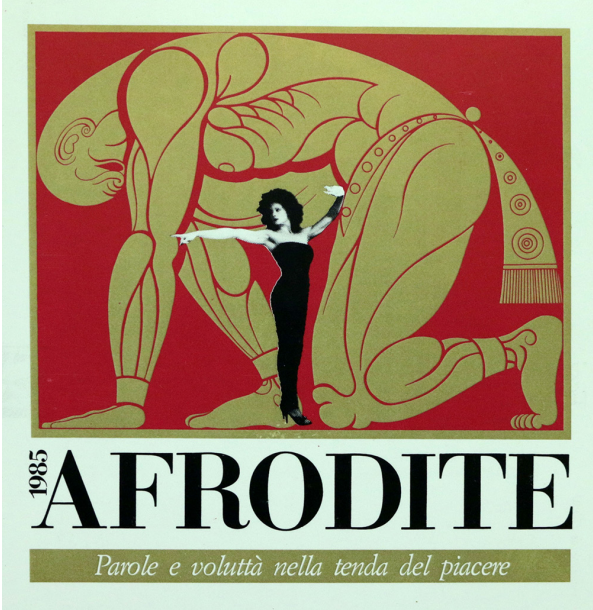

1985 - Programma delle iniziative presso lo spazio Fgci alla Festa dell'Unità di Modena ("Afrodite"). [art direction Elisabetta Ognibene, copy Francesco Ricci, Avenida] 


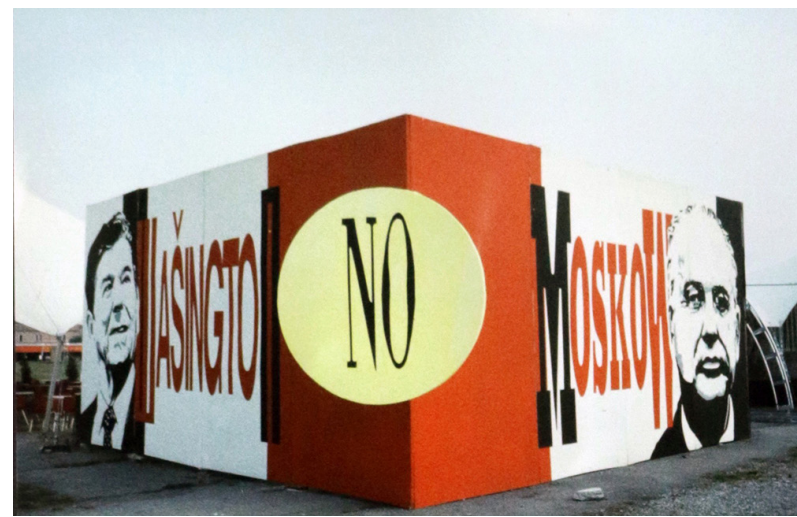

1986 - Allestimento dello spazio Fgci alla Festa dell'Unità di Modena ("No Washington, No Moscow"). [art direction Elisabetta Ognibene, copy Francesco Ricci, Avenida] creativi e grafici modenesi riuniti nello studio di progettazione "Kennedy's Studios". I materiali provenienti dagli eventi e dalle campagne promosse dai giovani comunisti modenesi mostrano un allontanamento progressivo ma radicale dai linguaggi appartenenti alla tradizionale propaganda di partito, in favore dell'introduzione di elementi visivi e

tematici provenienti dalle esperienze del mondo giovanile e dei nuovi movimenti sociali, ma anche dalla cultura di massa, emersi tra la fine degli anni Settanta e l'inizio degli anni Ottanta.

Il contesto in cui si realizzò questo scambio di idee e competenze appare, a posteriori, certamente favorevole a questo tipo di innovazione. Il 1983 viene solitamente citato come un anno di svolta per la comunicazione politica, soprattutto nell'ambito delle campagne elettorali: proprio nell' '83 gli spot elettorali televisivi fecero il loro debutto in Italia, mentre ormai quasi tutti i

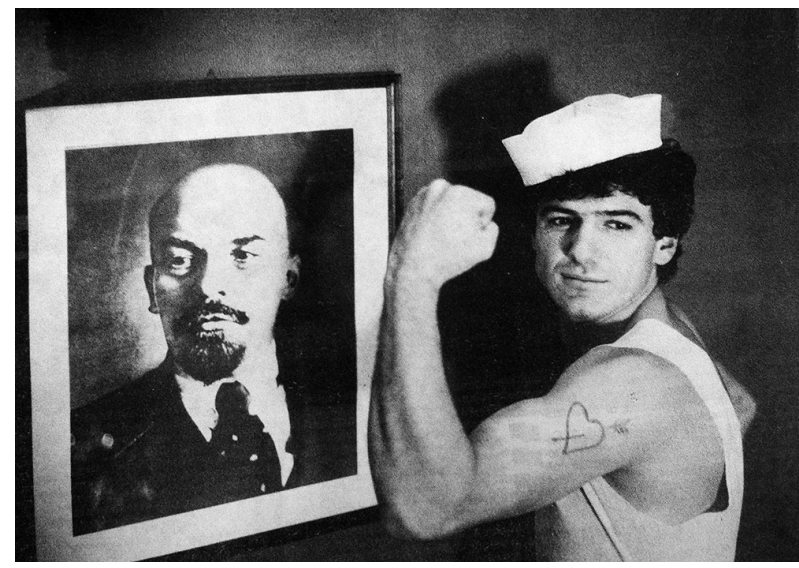

1986 - Dettaglio del servizio fotografico a corredo di uno degli articoli di "L'Assalto al Cielo", realizzato all'interno della sede della Fgci modenese. [art direction Elisabetta Ognibene/ Kennedy's studios] partiti avevano cominciato a servirsi di pubblicitari professionisti per curare i propri messaggi [Pezzini 2001]. Tuttavia, da questa tendenza generale rimaneva escluso uno dei protagonisti della politica parlamentare, che ancora per qualche anno si sarebbe rifiutato di seguire i propri avversari nella corsa alla spettacolarizzazione e alla cura dell'immagine del leader: il Partito comunista italiano [Cheles 2001]. La comu- 


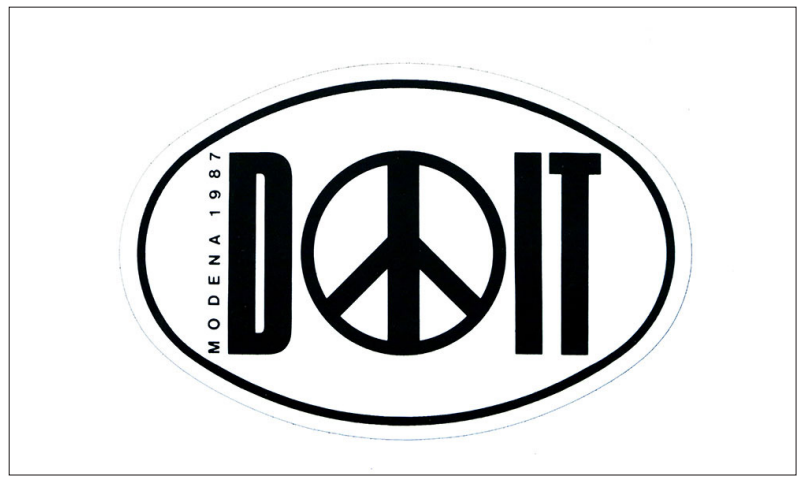

1987 - Adesivo con logo di "DOIT", rassegna di concerti organizzata dalla Fgci di Modena. [art direction Elisabetta Ognibene, copy Francesco Ricci, Avenida] nicazione politica, dunque, si delinea come una delle dimensioni chiave da cui partire per l'analisi di questo periodo, un terreno su cui lo scarto che in quel momento stava emergendo tra la spinta innovatrice della Fgci e l'immobilismo del Pci si fa più evidente.

È significativa, a questo proposito, la testimonian-

za dello storico britannico Stephen Gundle, che nell'introduzione al suo studio del rapporto tra Pci e mutamento culturale riporta le impressioni ricevute da una passeggiata tra gli stand della Festa dell'Unità provinciale di Modena del 1987. Gundle si sofferma prima ad ascoltare il discorso di un noto dirigente, che dal palco destinato ai dibattiti tuona contro la povertà culturale di spettacoli come il recente tour italiano della pop star Madonna, e approda poi allo spazio gestito dalla Fgci, che gli offre invece questa immagine:

Su uno dei pannelli campeggiava la scritta "Fondazione degli amici dello Stato di Strelax" cui seguiva un elenco semiserio di idoli ed eroi: il giovane Holden, Sergio Leone, John Ford, Vladimir Majakovskij, gli ammutinati del Bounty, la redazione di "Di tasca nostra", l'associazione "Italia nostra", Brigitte Bardot, Winnie e Nelson Mandela, Sandro Pertini, Sacco e Vanzetti, il Che, Beppe Grillo, Mary Poppins, Carlo Pisacane, il Club alpino italiano, i Beatles, Toro Seduto, i Three Stooges, Corto Maltese, Charlie Chaplin, Rosa Luxemburg e Gigi Riva. [Gundle 1995, 4]

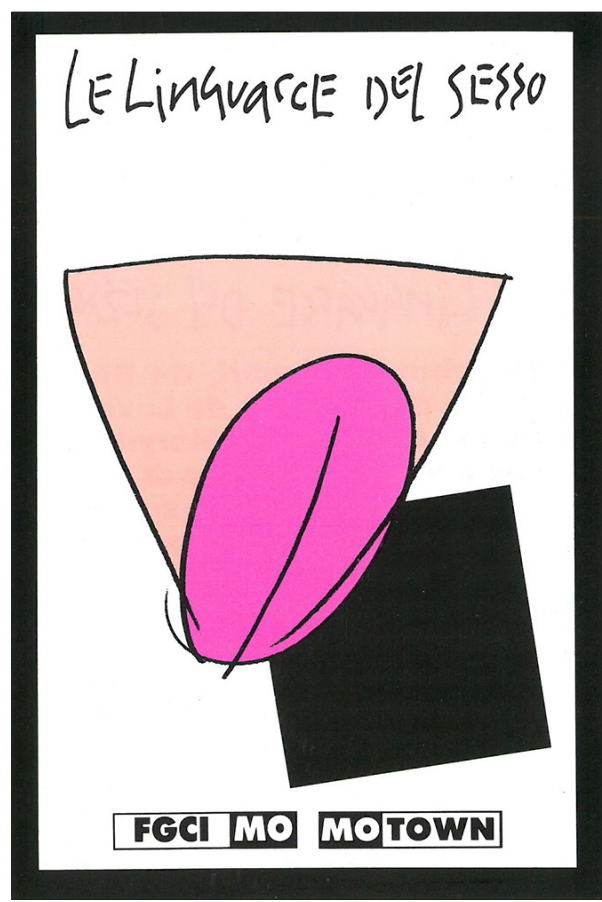

1989 - Programma dell'iniziativa "Le linguacce del sesso", serata organizzata dalla Fgci modenese dedicata a giovani e sessualità. [art direction Elisabetta Ognibene, copy Francesco Ricci, Avenida 


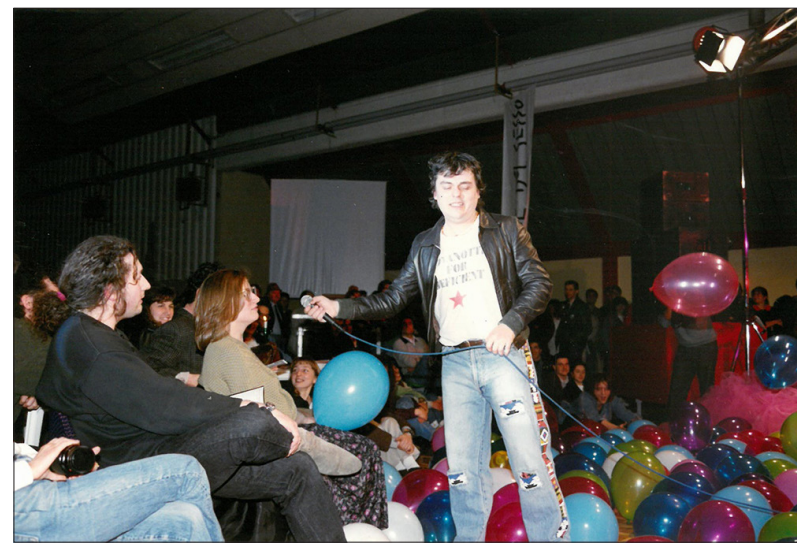

1989 - Roberto "Freak" Antoni interviene durante l'iniziativa "Le linguacce del sesso". [art direction Elisabetta Ognibene, copy Francesco Ricci, Avenida]
Come è facile cogliere da questo piccolo quadro, la dialettica tra passato e presente, tra linea tradizionale e rinnovamento, era uno dei nodi più importanti del dibattito aperto dalla rifondazione della Fgci a metà degli anni Ottanta. Il gruppo di grafici modenesi dei "Kennedy's Studios" e la dirigenza della Fgci provinciale affrontarono questo dualismo attraverso un nuovo approccio ai linguaggi della politica, che fondeva simboli e parole chiave appartenenti alla tradizione del partito con nuovi temi e repertori simbolici provenienti da altre storie e da altre esperienze. Il successo di questo esperimento è testimoniato anche dal fatto che, nel giro di pochi anni, gli stessi creativi modenesi passarono a curare la comunicazione della Fgci nazionale e, nei primi anni dopo lo scioglimento del Pci, della nuova Sinistra Giovanile.

\section{Prime conclusioni e prospettive di interpretazione}

Il caso della Fgci modenese, allora, non è solo interpretabile nel contesto del passaggio da propaganda a comunicazione politica che molti partiti avevano intrapreso all'inizio degli anni Ottanta, ma può essere considerato anche come un esempio di innovazione simbolica, un processo tipico dei periodi di trasformazione delle culture politiche [Harrison 1995]. La scelta di mescolare repertori simbolici differenti e, di conseguenza, rimodulare

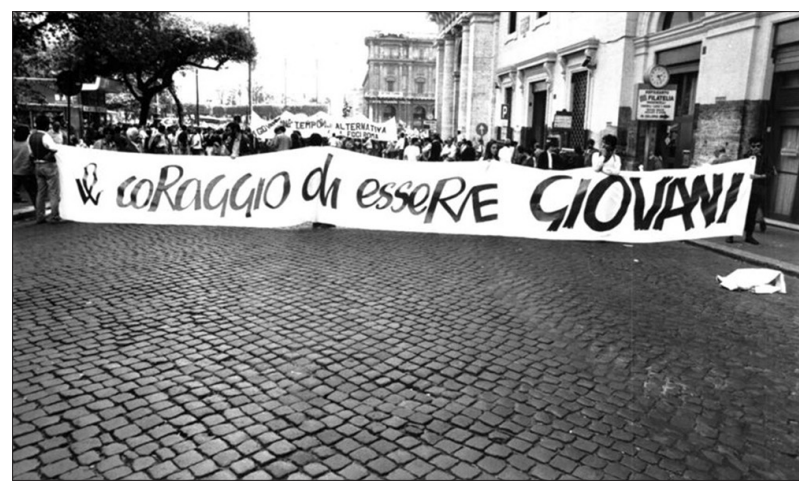

1989 - Manifestazione nazionale Fgci, 6 maggio, Roma. [art direction Elisabetta Ognibene/ Avenida] 
l'identità politica comunista con l'introduzione di nuove istanze e obiettivi, era una risposta alla crisi generale del comunismo italiano che di fatto precedeva il dibattito che avrebbe poi accompagnato la svolta occhettiana dell'1989-1991. Tuttavia, è proprio il rapporto sottostante con il bagaglio mnemonico e simbolico del Pci a differenziare questi due tentativi: mentre con lo scioglimento del Pci si decise di imporre una forte discontinuità con il passato attraverso l'abbandono dell'intero apparato simbolico legato al partito, alla base dell'esperimento della Fgci modenese era evidente la volontà di mantenere un legame con il proprio patrimonio, rinnovandone forme e contenuti [Kertzer 1996].

La trasformazione dell'organizzazione e le nuove proposte della dirigenza Fgci suggeriscono così che, diversamente da quanto generalmente proposto dalla letteratura sul periodo, si possa in effetti parlare della nascita di una nuova generazione politica di militanti, attivisti e dirigenti che durante gli anni Ottanta fornì un contributo originale alla cultura e alla politica comunista italiana. La ricostruzione di questa stagione politica, dunque, costituisce un tassello indispensabile per comporre un'immagine completa di questo decennio, introducendo elementi di problematizzazione inediti e, allo stesso tempo, aprendo nuove linee di riflessione sulla scomparsa della cultura politica comunista italiana nata nel secondo dopoguerra.

\section{Bibliografia}

Barbagli M. e Corbetta P. 1980, L'elettorato, l'organizzazione del Pci e i movimenti, "il Mulino", 3

Cheles L. 2001, Picture Battles in the Piazza: The Political Poster, in Cheles L. e Sponza L. (eds.) 2001, Political Communication in Italy from 1945 to the 1990s, Manchester: Manchester University Press

Crainz G. 2012, Il paese reale. Dall'assassinio di Moro all'Italia di oggi, Roma: Donzelli Editore

Flores M. e Gallerano N. 1992, Sul Pci. Un 'interpretazione storica, Bologna: il Mulino

Fotia C. 1985, Il Pci sgrida i suoi ragazzi, "Il manifesto", 23 febbraio

Ginsborg P. 1998, L'Italia del tempo presente, Torino: Einaudi

Gundle S. 1995, I comunisti italiani tra Hollywood e Mosca. La sfida della cultura di massa (1943-1991), Firenze: Giunti

Harrison S. 1995, Four Types of Symbolic Conflict, "The Journal of the Royal Anthropological Institute", 1(2)

Hellman S. 1980, Il Pci e l'ambigua eredità dell'autunno caldo a Torino, "il Mulino”, 2 
Kertzer D.I. 1996, Politics and Symbols: The Italian Communist Party and the Fall of Communism, New Haven: Yale University Press

Ignazi P. 1992, Dal Pci al Pds, Bologna: il Mulino

Lange P., Irvin C. e Tarrow S. 1990, Mobilization, Social Movements and Party Recruitment: The Italian Communist Party Since the 1960s, "British Journal of Political Science", 20(1)

Mannheimer R. 1990, Vecchi e nuovi caratteri del voto comunista, in Caciagli M. e Spreafico A. (eds.) 1990, Vent'anni di elezioni in Italia: 1968-1987, Padova: Liviana

Mori M.A. 1988, Molti gli iscritti, pochi i voti ecco il paradosso della Fgci, "La Repubblica", 9 dicembre

Pezzini I. 2001, Advertising Politics on Television: The Party Election Broadcast, in Cheles L. e Sponza L. (eds.) 2001, Political Communication in Italy from 1945 to the 1990s, Manchester: Manchester University Press 